\title{
LA APLICACIÓN DE DATOS FAUNÍSTICOS PARA EL DISEÑO DE REDES DE RESERVAS: EL CASO DE LOS ANFIBIOS Y REPTILES DE LA PENÍNSULA IBÉRICA
}

\author{
J. M. Lobo ${ }^{1}$ y M. B. Araújo²
}

\begin{abstract}
RESUMEN
A pesar de que disponemos de herramientas de selección de reservas que permiten maximizar la representación de las especies con un mínimo coste, las decisiones sobre la ubicación de nuestra red de espacios protegidos siguen realizándose todavía sin el concurso de estas técnicas. En este trabajo se estudian y comparan los resultados de estrategias distintas de selección de reservas (puntos de máxima riqueza, puntos de máxima rareza y solución de máxima cobertura), estimando su efectividad a la hora de representar la diversidad de anfibios y reptiles ibéricos. Los resultados obtenidos han sido comparados, tanto con una selección al azar, como con una selección que considera la ubicación actual de las reservas. Los espacios naturales protegidos no permiten representar la diversidad de anfibios y reptiles ibéricos mejor de lo que lo haría una selección de reservas al azar, mientras que la solución de máxima cobertura permite obtener niveles de representación de las especies mucho mayores que cualquier otra estrategia. Sería recomendable que los gestores ambientales seleccionaran las áreas de conservación utilizando los eficaces algoritmos de selección disponibles. Si no se hace así, corremos el riesgo de que la inversión en conservación de nuestros limitados recursos produzca una baja rentabilidad.
\end{abstract}

Palabras clave: selección de reservas, Península Ibérica, Anfibios, Reptiles.

\section{ABSTRACT \\ The use of faunistic data for the design of reserve networks: the case of the amphibians and reptiles in the Iberian Peninsula}

Real-world reserve selection decisions are still performed without use of available reserve selection tools that maximise conservation benefit for a minimum cost. In this paper we investigate the consequences of using different reserve selection strategies (richness hotspots, rarity hotspots and maximum covering solutions) to represent amphibian and reptiles species diversity in the Iberian Peninsula. We compare results with that observed in current reserve networks and that expected by chance. We found that current reserves conserve fewer amphibian and reptiles species than expected by chance and that great improvements could be achieved if maximum covering solutions were used to support reserve selection decisions. We recommend that conservation planners and other decision makers use available knowledge and technology for the selecting of important areas for biodiversity. Otherwise, there is a risk that limited resources are invested in such a way as to provide poor conservation return.

Key words: Reserve selection, Iberian peninsula, Amphibian, Reptiles.

Departamento de Biodiversidad y Biología Evolutiva, Museo Nacional de Ciencias Naturales, c/ José Gutiérrez Abascal 2. 28006, Madrid, España

2 Macroecology and Conservation Unit, University of Évora, 7000-730 Évora. Portugal. Centre d'Ecologie Fonctionnelle et Evolutive, CNRS 34293, Montpellier, France. 


\section{Introducción}

Ante la dificultad de modificar nuestra gestión ambiental a lo largo de un territorio, nuestra política de conservación se basa, principalmente, en la creación de santuarios o espacios protegidos en diverso grado de nuestra adversa influencia. Los criterios para la selección de estos espacios suelen ser muy variables y, en ocasiones, subjetivos. En el mejor de los casos, la opción de selección busca maximizar la variabilidad ambiental del territorio. Aunque en la práctica y por diversos motivos (Prendergast et al., 1999) no sean utilizados por los gestores medioambientales (ver por ejemplo,

http://europa.eu.int/comm/environment/nature/home.htm, http://www.europarc-es.org/ y

http://www.iambiente.pt/pls/ia/homepage)

existen algoritmos que permiten seleccionar un conjunto de áreas ambientalmente representativas a lo largo de un gradiente ambiental, con la intención de que ese conjunto de áreas sea capaz de representar la diversidad biológica de un territorio (Faith \& Walter, 1996). Sin embargo, el supuesto de que la variabilidad ambiental puede tomarse como un sustituto de la diversidad biológica no está empíricamente demostrado. Utilizando diversa información ambiental y los datos disponibles sobre la distribución geográfica de los vertebrados terrestres, Araújo et al. (2001) encuentran que la variabilidad ambiental predice mal la diversidad de especies en Europa. Ello es así, probablemente, porque otros tipos de factores histórico-geográficos únicos e irrepetibles (Ricklefs \& Schluter, 1993) condicionan la distribución de los organismos, produciendo que áreas ambientalmente similares alojen conjuntos de especies diferentes.

Si la utilización de la variabilidad ambiental como criterio de selección de las áreas protegidas para la conservación de la diversidad biológica es inadecuada, también lo es frecuentemente el uso de las especies emblemáticas (Simberloff, 1998; Williams et al., 2000), o la simple consideración de las áreas ricas en especies o con más especies raras (Pressey et al., 1993; Williams et al., 1996; Reyers et al., 2000; Turpie et al., 2000). Ello se debe a la habitual ausencia de covariación espacial entre las especies y a que la riqueza de especies o la rareza son parámetros que no contemplan la composición específica y singular de cada localidad. De este modo, para ser fiable, el diseño de una red de espacios protegidos con capacidad para conservar la diversidad biológica debe basarse en la propia información faunística y florística y, cuando nues- tro conocimiento sobre la distribución esté espacialmente sesgado, en los resultados de modelos predictivos de distribución elaborados a partir de la información corológica de buena calidad disponible (Andriamampianina et al., 2000; Araújo \& Williams, 2000). En este trabajo se utiliza, como ejemplo, la información corológica disponible sobre la distribución de los anfibios y reptiles en la Península Ibérica en cuadrículas UTM de 50 x 50 $\mathrm{km}$ para desarrollar un proceso de selección que identifique las áreas prioritarias de conservación para estos grupos. Los resultados obtenidos nos han permitido realizar una comparación preliminar de las propuestas de áreas de conservación según diversos criterios de selección, y estimar la efectividad de los actuales espacios naturales protegidos (ENP) para acoger la diversidad biológica de estos organismos. Por último, se comentan algunos de los subsiguientes pasos analíticos que sería necesario acometer, para aprovechar las posibilidades que tienen este tipo de procesos automatizados de selección de reservas.

\section{Métodos}

Los datos de los anfibios han sido tomados de Salvador \& García-París (2001), Gasc et al. (1997), Pleguezuelos (1997) y Pleguezuelos et al. (2002). Los datos de los reptiles fueron tomados de Pleguezuelos et al. (2002) complementados con los de Salvador (1998) cuando existía información adicional. En total se han considerado 27 especies de anfibios y 43 de reptiles. Se han excluido las islas Columbretes y Alboran, así como las especies o poblaciones de introducción antigua o reciente y las traslocaciones (Pleguezuelos, 2002).

Para el total de las cuadrículas UTM IberoBaleares de 50 x $50 \mathrm{Km}$. consideradas $(\mathrm{n}=257$; ver Figura 1), hemos tomado como valor umbral el diseño de un red de reservas que abarcase, como máximo, el $10 \%$ de total de las cuadrículas $(n=26)$ y para esa cifra se ha estimado cual es el grado de representación máximo de las especies de anfibios y reptiles que puede alcanzarse. La solución óptima (solución de máxima cobertura o maximal-covering set of areas) se ha obtenido utilizando el algoritmo diseñado por Margules et al. (1988) y modificado por Williams (1999), el cual maximiza la representación de las especies para un número determinado de áreas. Este algoritmo utiliza el criterio de complementariedad, seleccionando aquel conjunto de áreas que, conjuntamente, posee la mayor riqueza de especies. Es decir, busca áreas que, secuencial- 


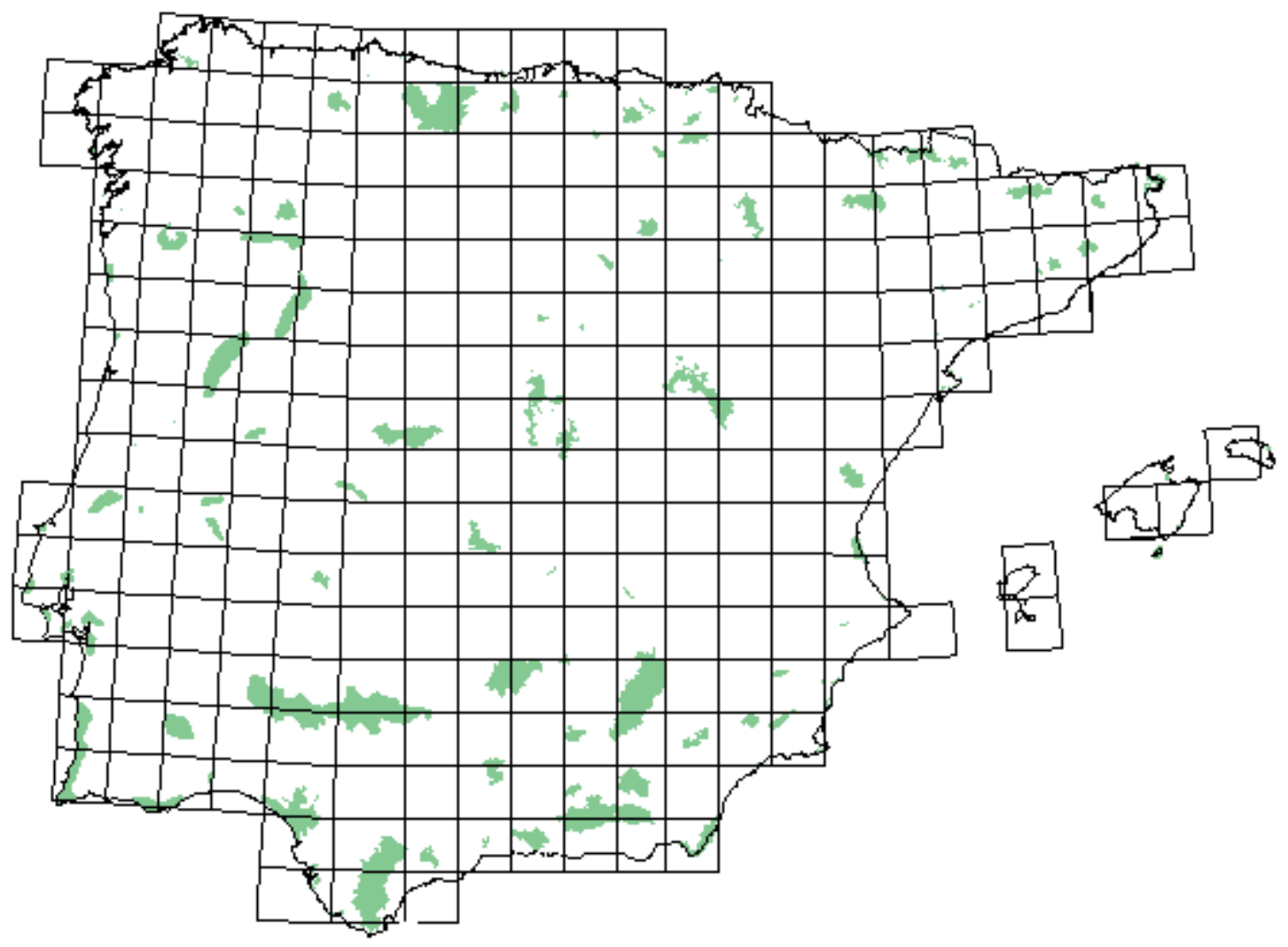

Fig. 1.-Cuadrículas UTM de 50x $50 \mathrm{Km}$. consideradas en este estudio y localización de los espacios naturales protegidos (ENPs).

Fig. 1.- Considered 50 x $50 \mathrm{~km}$ UTM squares and location of the natural protected areas (ENPs)

mente, incluyen un mayor número de especies previamente no representadas. Como los algoritmos de selección basados en la rareza se han mostrado más eficaces (Kershaw et al., 1994, Csuti et al., 1997), se eligen primero aquellas cuadrículas con especies raras (las presentes en una única cuadrícula, por ejemplo) para, secuencial e iterativamente, ir eligiendo aquellas otras complementariamente más ricas en especies en la que se incluya la especie más rara aún no representada.

Para comprobar si la representación óptima obtenida difiere de una selección aleatoria, el mismo número de cuadrículas $(n=26)$ fue seleccionado al azar 1000 veces calculando el porcentaje de especies representadas y estimando el límite de confianza de dicho porcentaje al 95\%. Junto a estas dos selecciones (óptima y aleatoria) se identificaron las 26 cuadrículas con mayor riqueza en especies (hotspots of richness) y las 26 con mayor rareza (hotspots of rarity), estimando esta última como el sumatorio de los valores de rareza individuales de cada una de las especies presentes en una cuadrícula $\left(\sum R_{i}\right)$, en donde $R_{i}$ es el inverso del número de cuadrículas ocupadas por una especie (ver Kier \& Barthlott, 2001).

Para estimar la capacidad de los espacios naturales protegidos (ENPs, ver Figura 1) a la hora de representar las especies de anfibios y reptiles ibéricos, se ha calculado el porcentaje que suponen los ENPs en cada una de las cuadrículas IberoBaleares sobre el total de la superficie terrestre de esas cuadrículas. Teniendo en cuenta esas cifras se delimitaron aquellas cuadrículas con una superficie de ENPs mayor o igual al 5\% $(n=78)$ y al $20 \%(n=28)$ de su superficie, estimando el porcentaje de especies representadas en estas cuadrículas y comparándolo con una selección de cuadrículas al azar.

Todos los cálculos han sido efectuados mediante el software WORLDMAP (Williams, 1999). 
$\mathbf{A}$

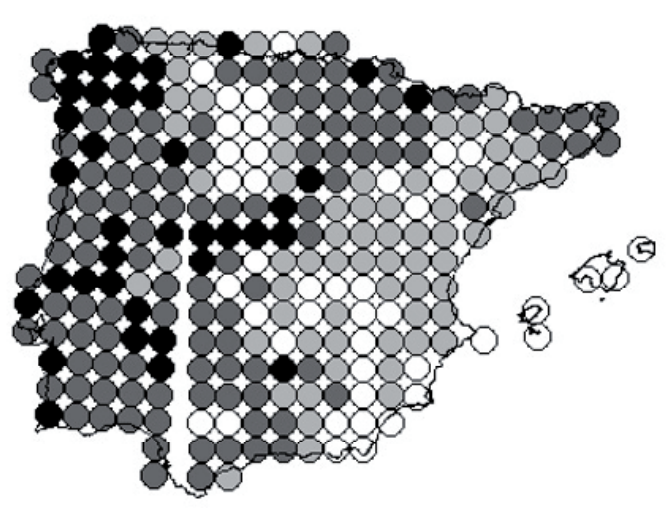

C

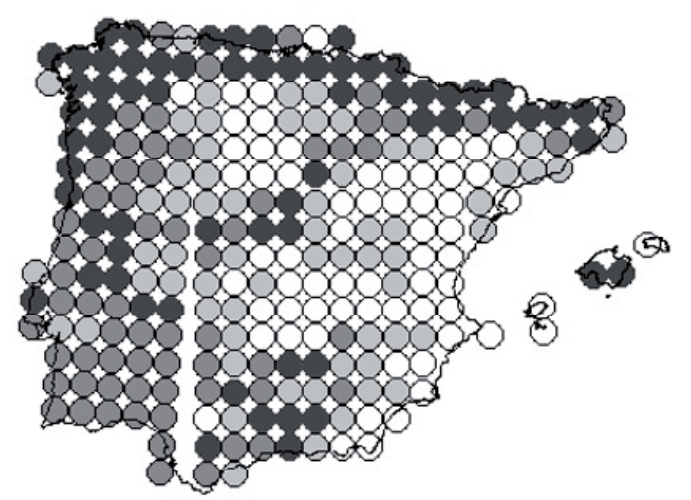

B

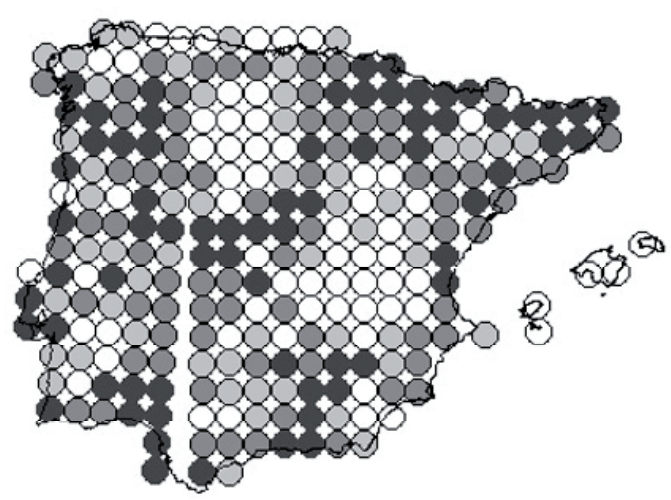

D

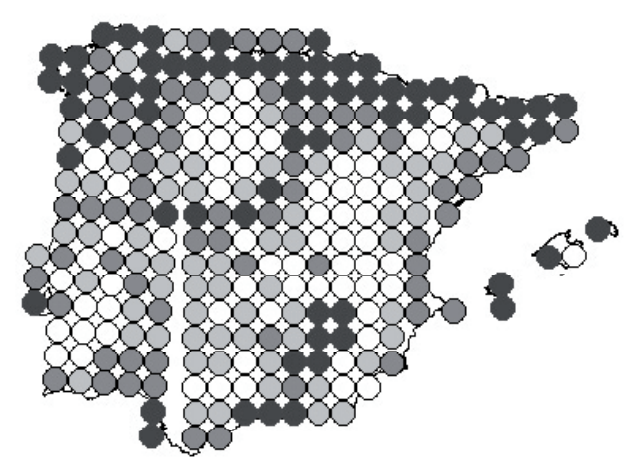

Fig. 2.- Variación geográfica de la riqueza de especies de anfibios (A) y de reptiles (B) en la Península Ibérica, y de la rareza de anfibios (C) y de reptiles (D). Los valores se han dividido en cuatro cuartiles que oscilan entre el negro (las cuadrículas con mayor riqueza o rareza) y el blanco.

Fig. 2.- Geographic variation of amphibian (A) and reptile (B) Iberian species richness, and amphibian (C) and reptile (D) range size rarity. The values have been divided in four quartile that oscillate between the black (the richest cells o with more rare species) and the white ones.

\section{Resultados}

\section{SELECCIÓN DE ÁREAS DE CONSERVACIÓN}

La distribución geográfica de la riqueza de anfibios sigue, a grandes rasgos, un patrón longitudinal con las zonas más ricas claramente ubicadas al oeste de la Península Ibérica. El patrón de variación espacial de la riqueza de reptiles en más complejo, con bastantes zonas ricas en especies situadas áreas montañosas. La rareza en ambos grupos varía, sin embargo, de un modo similar, con mayores valores de rareza en todo el norte peninsular, pero también en las montañas centrales y del sureste así como en las Baleares (Figura 2).

La selección del 10\% de las cuadrículas de acuerdo a la riqueza de especies permite representar el $96 \%$ de las especies de anfibios y el $88 \%$ de las especies de reptiles Ibero Baleares. La selección de áreas teniendo en cuenta la rareza ofrece mejores resultados, permitiendo incorporar el $100 \%$ de los anfibios y el $98 \%$ de los reptiles, aproximadamente (Tabla 1). Una selección al azar de las cuadrículas permite siempre una alta representación de las especies que no difiere significativamente $(\mathrm{P}<0,05)$ de la 
Tabla 1.- Porcentaje de representación de las especies de anfibios y reptiles Ibéricas de acuerdo a diferentes estrategias de selección. Se ha seleccionado el 10\% de las cuadrículas UTM Ibéricas de 50 x $50 \mathrm{~km}(n=26)$ al azar (1000 replicaciones) y de acuerdo a su riqueza de especies (S), su rareza (R) o la solución de máxima cobertura ("maximum coverage set solution"). Por otra parte, se ha calculado el porcentaje de especies representadas por él número de cuadrículas con más del $5 \%(\mathrm{n}=78)$ y del $20 \%$ $(\mathrm{n}=28)$ de su superficie cubierta por espacios naturales protegidos $($ ENP) y el porcentaje de especies representado por una selección aleatoria (1000 replicaciones) de ese mismo número de cuadrículas. * Solo 5 cuadrículas son necesarias para representar el $100 \%$ de las especies y 28 cuadrículas permiten siete representaciones de cada especie. ** Solo 9 cuadrículas son necesarias para representar el 100\% de las especies y 30 cuadrículas permiten cinco representaciones de cada especie.

Table 1.- Percentage representation of Iberian amphibian and reptile species with different reserve selection strategies. The $10 \%$ of 50 x $50 \mathrm{~km}$ Iberian UTM squares $(n=26)$ were selected at random (1000 replications) and according to its species richness $(\mathrm{S})$, its rarity $(\mathrm{R})$ and the maximum coverage set solution. The percentage of species represented by the squares with more than $5 \%$ or $20 \%$ of their area protected (ENP) was also calculated ( $n=78$ and $n=28$, respectively), as well as the percentage of species represented by a random selection of the same number of squares (1000 replications). * Only 4 squares needed for $100 \%$ representation and 28 squares allow 7 representations of all species. ** Only 9 squares needed for $100 \%$ representation and 30 squares allow 5 representations of all species.

\begin{tabular}{lccc}
\hline & $\mathbf{N}^{\mathbf{0}}$ de áreas & Anfibios & Reptiles \\
\hline Al azar (random) & 26 & $92,31 \%$ & $81,40 \%$ \\
Riqueza de especies (S) & 26 & $96,15 \%$ & $88,37 \%$ \\
Rareza (R) & 26 & $100 \%$ & $97,67 \%$ \\
Solución de máxima cobertura & 26 & $100 \% \times 7 *$ & $100 \% \times 5 * *$ \\
ENP áreas (>20\%) & 28 & $88,46 \%$ & $83,72 \%$ \\
Al azar (random) & 28 & $90,31 \%$ & $83,72 \%$ \\
ENP áreas (>5\%) & 78 & $96,15 \%$ & $93,02 \%$ \\
Al azar (random) & 78 & $100 \%$ & $97,67 \%$ \\
\hline
\end{tabular}

que puede obtenerse mediante una selección de acuerdo a la riqueza de especies de reptiles o anfibios, o la rareza de anfibios. En el caso de la rareza de los reptiles, una selección al azar de las cuadrículas permite representar un número significativamente menor de especies (Tabla 1). Las cuadrículas seleccionadas según la riqueza de especies se localizan principalmente en el cuadrante noroccidental peninsular, para los anfibios, y en la costa catalana, el sistema central y algunas áreas meridionales en el caso de los reptiles. Las cuadrículas seleccionadas según la rareza siguen un patrón de localización relativamente similar en ambos grupos, patrón que está íntimamente relacionado con la variación geográfica de la rareza (Figura 3 ).

La solución de máxima cobertura permite representar el $100 \%$ de las especies de anfibios con solo 5 cuadrículas y el $100 \%$ de las especies de reptiles con 9 , pero además, la elección del $10 \%$ de las cuadrículas Íbero Baleares con este criterio asegura entre cinco y siete representaciones de cada especie, aproximadamente (Tabla 1). Las cuadrículas seleccionadas mediante este criterio en el caso de los anfibios, se localizan prioritariamente en los Pirineos occiden- tales y en las islas Baleares (debido a la presencia de Alytes muletensis). Otras áreas con valor de conservación se ubican en la Cornisa Cantábrica, el Sistema Penibético y la costa Atlántica y el Ribatejo portugués. En el caso de los reptiles, las cuadrículas seleccionadas se localizan en los Pirineos centrales y orientales, las islas Baleares, el extremo noroccidental de la costa Cantábrica, el sur del Sistema Penibético, el delta del Guadalquivir y, en menor medida, la Serrania de Cazorla (Figura 4).

\section{EFICIENCIA DE LOS ENPS}

Seleccionar la totalidad de las cuadrículas con más del $20 \%$ de su superficie terrestre protegida $(\mathrm{n}=28)$ permite representar, aproximadamente, el $88 \%$ y el $84 \%$ de las especies de anfibios y reptiles, respectivamente. Estos porcentajes son iguales o significativamente menores que los se podría obtener si ese mismo número de cuadrículas fuera seleccionado al azar $(\mathrm{P}<0,05$; Tabla 1$)$. Si somos menos exigentes y consideramos todas las cuadrículas con más del $5 \%$ de su superficie terrestre protegida $(n=78)$, los porcentajes de especies representadas aumentan hasta el $96 \%$ en el caso de 

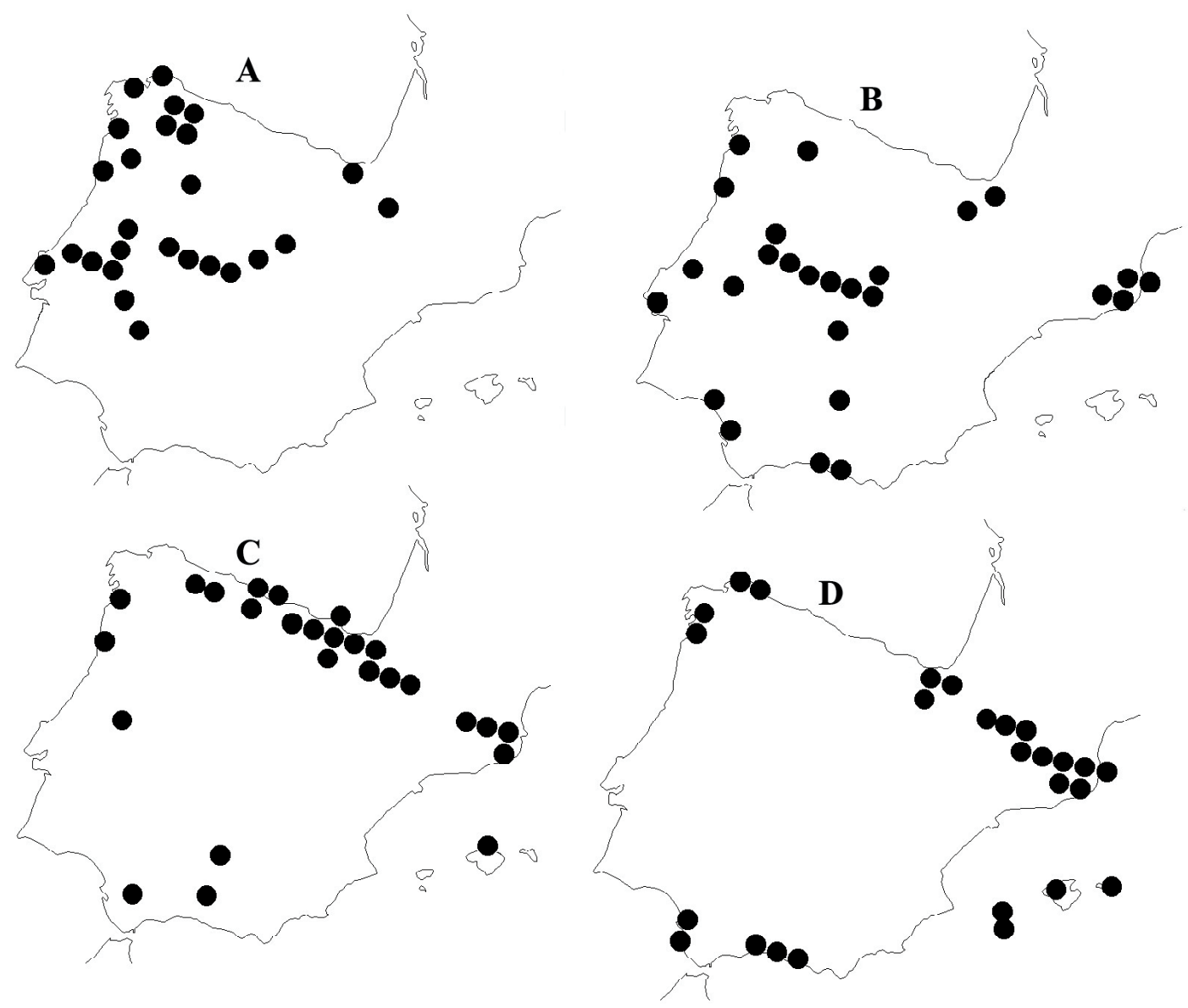

Fig. 3.- Cuadrículas UTM de 50 x $50 \mathrm{Km}$. seleccionadas de acuerdo a la riqueza de anfibios (A) y reptiles (B), y a la rareza de anfibios (C) y reptiles (D). El número de cuadrículas seleccionadas corresponde al $10 \%$ del total $(\mathrm{n}=26)$

Fig. 3.- Selected $50 \times 50 \mathrm{~km}$ UTM squares according to amphibian (A) and reptile (B) species richness scores, and amphibian (C) and reptile (D) rarity scores. The number of selected squares is the $10 \%$ of total $(n=26)$.

los anfibios y el $93 \%$ en los reptiles, pero tampoco podemos obtener una mejor representación de estos grupos que la podría conseguirse si las cuadrículas fuesen seleccionadas al azar (Tabla 1). Los territorios en donde se encontrarían las especies no representadas por los ENPs se sitúan, básicamente, en los Pirineos y en las islas Baleares (Figura 5). De este modo, considerando únicamente aquellos ENPs con más de $125 \mathrm{~km}^{2}$ de superficie, parece que no es posible garantizar la representación de la totalidad de las especies de anfibios y reptiles Íbero Baleares y que incluso una selección aleatoria de las reservas ofrecería una mejor cobertura de protección (Figura 6).

\section{Discusión}

Como ya ha sido demostrado en otras ocasiones (Pressey et al., 1993; Williams et al., 1996), la selección de áreas de conservación considerando la riqueza de especies o la rareza no es capaz de producir resultados fiables. Una delimitación de áreas de conservación basada en la riqueza apenas es capaz de representar las especies de anfibios y reptiles ibéricas mejor de lo que lo haría una selección al azar y, aunque las elecciones basadas en la rareza son mas eficaces, la mejor aproximación consiste en utilizar todo el conjunto de información corológica disponible sobre las especies y un algoritmo 

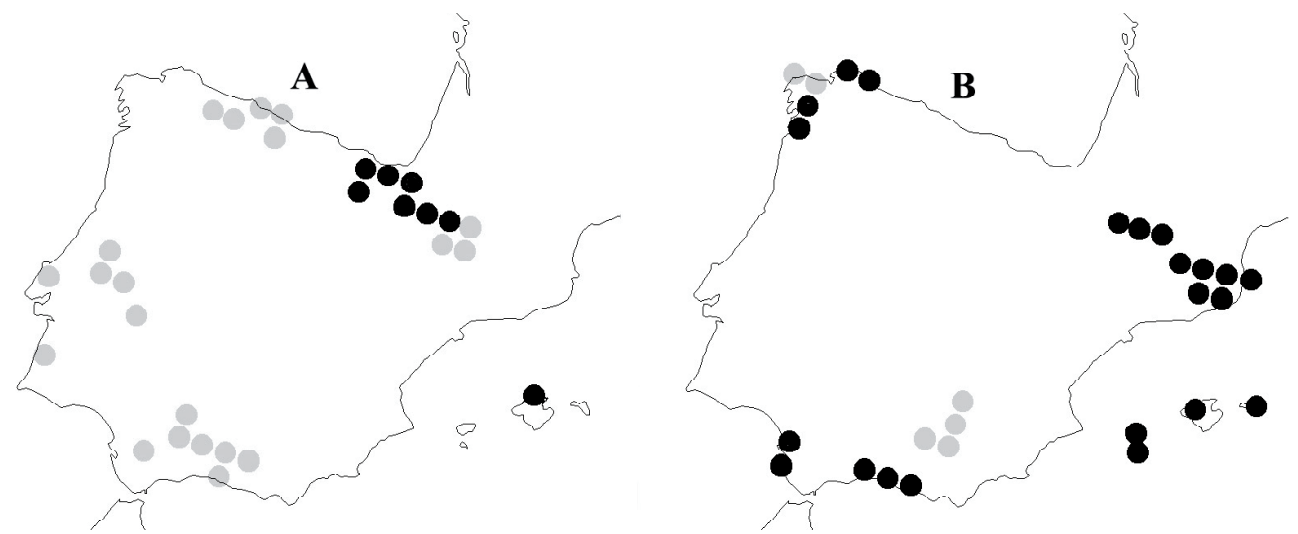

Fig. 4.- Solución de máxima cobertura para los anfibios (A) y los reptiles (B) considerando el 10\% de las cuadrículas Ibéricas (ver métodos y Margules et al., 1988). Las cuadrículas negras son áreas irremplazables (en las que existe una especie que solo aparece en esa cuadrícula) mientras que las grises (cuadrículas flexibles) han sido elegidas entre otras posibilidades y, por tanto, pueden variar en su localización según las posibilidades del diseño.

Fig. 4.- Maximal-covering set of areas for amphibians (A) and reptiles (B) for a $10 \%$ of Iberian squares (see methods and Margules et al., 1988). Black circles are irreplaceable areas (where a species that only occur in one square is present) while grey circles (flexible areas) are selected between other possibilities, and can vary in their localization according to the possibilities of the design.
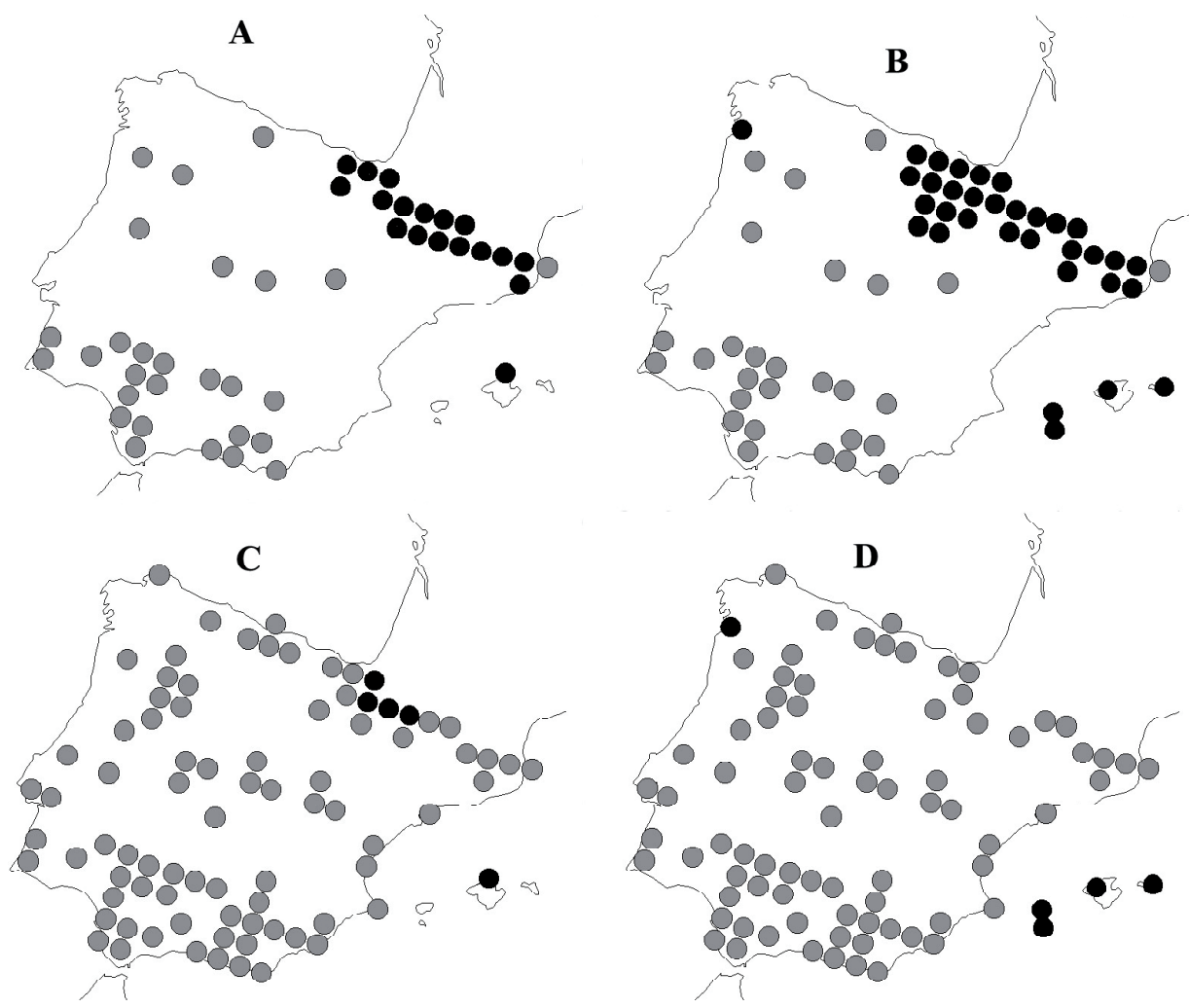

Fig. 5.-Cuadrículas seleccionadas entre aquellas con más del $20 \%$ de su superficie terrestre protegida $(n=28)$ para los anfibios (A) y los reptiles (B), y cuadrículas seleccionadas con mas del 5\% de su superficie protegida $(n=78)$ para los anfibios (C) y los reptiles (D). Los círculos más oscuros son las cuadrículas UTM en donde se encuentran especies no representadas.

Fig. 5. - Selected Iberian squares between those with more than $20 \%$ of its area protected $(n=28)$ for amphibians $(A)$ and reptiles (B), and selected squares with a protected area higher than 5\% for amphibians (C) and reptiles (D). Black circles are UTM squares where unrepresented species occur. 
A

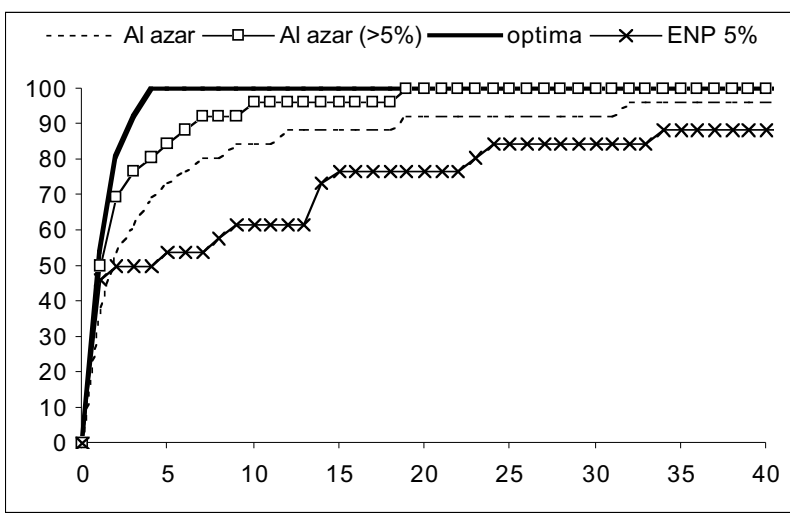

B

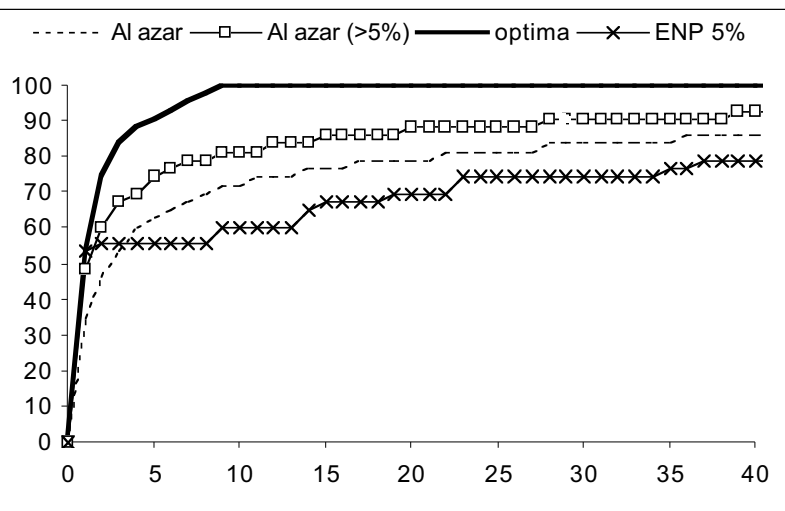

Fig. 6.- Variación del porcentaje de especies de anfibios (A) y reptiles (B) representadas sobre el total Ibérico con el incremento en el número de cuadrículas UTM de 50 x $50 \mathrm{Km}$. seleccionadas (eje de las X) de acuerdo a distintos criterios. La línea continua representa la solución optima (en este caso el mínimo número de áreas requerido para una completa representación de todas las especies, ver Margules et al., 1988). La línea discontinua es la representación media obtenida de una selección al azar (1000 repeticiones), mientras que la línea inmediatamente por encima es el límite de confianza superior al 95\%. ENPs es el porcentaje de especies representado por una selección de las cuadrículas con mas del $5 \%$ de su superficie protegida $(n=78)$.

Fig. 6.- Variation in percentage of represented Iberian amphibian (A) and reptiles (B) species with the increment in the number of 50 x 50 UTM squares (X axis), according to different criteria. The continuous line is the optimal solution (in this case the minimum set of areas required to represent all species, see Margules et al., 1988). Broken line represents the result of a random selection (1000 replicates), while the line located above is the $95 \%$ confidence limit. ENPs line is the percentage of species represented by a selection of squares with a protected area higher than $5 \%(n=78)$.

de selección que tenga en cuenta el criterio de complementariedad. Entre las ventajas de estas técnicas, es necesario recalcar la posibilidad de repetir los análisis temporalmente y evaluar, con criterios contrastables y eficaces, el diseño de la red de reservas que se efectúe. Otra ventaja no menos importante de estos métodos de selección basados en la complementariedad es que, si por variadas razones, la solución obtenida es irrealizable, es posible buscar selecciones alternativas de áreas que no disminuyan la representación de las especies.

Hasta ahora, los análisis de selección de reservas para la conservación de los anfibios y reptiles ibéricos se han basado en la simple consideración de variables sinecológicas como la riqueza de especies o la rareza (Montaña \& Rey Benayas, 2002), o en la utilización conjunta de estas variables y las categorías de conservación (Mateo, 2002; ver, sin embargo, Araújo, 1999). Con ser muy loables, estos acercamientos a la delimitación de las áreas de conservación resultan insuficientes. Como hemos visto en el caso de los anfibios y reptiles ibéricos, una selección circunscrita a aquellas cuadrículas con más de $125 \mathrm{~km}^{2}$ protegidos no es mejor que una selección aleatoria. Aunque es evidente que deben analizarse también aquellos ENPs con una menor superficie, este resultado demuestra la necesidad de considerar los datos faunísticos cuando delimitemos una red de espacios protegidos. En otras palabras, diseñar redes de reservas eficaces para la protección de los "actores" de los ecosistemas, requiere utilizar información faunística y abandonar la idea simplista de que una selección que represente la variabilidad ambiental, garantiza la conservación de la diversidad biológica.

Los resultados obtenidos en este trabajo deben considerarse un ejemplo de la aplicación de estas técnicas, un primer acercamiento elemental que debería mejorarse con información faunística a menor resolución espacial y datos biológicos más precisos. Evidentemente, una resolución espacial como la utilizada en este trabajo (cuadrículas de $2.500 \mathrm{~km}^{2}$ ) produce resultados groseros a la hora de delimitar la ubicación de los espacios protegidos capaces de representar la diversidad biológica de un grupo taxonómico dado. Por ello, la localización concreta de las áreas a proteger debe, posteriormente, perfeccionarse mediante el análisis a menor esca- 
la dentro de cada cuadrícula o región (Margules \& Pressey, 2000). Esto es especialmente cierto en el ejemplo analizado en este trabajo, ya que las localidades concretas para la pervivencia de algunas especies pueden ser áreas de muy escasa superficie. En ocasiones, si la resolución y la extensión de la información corológica no es suficiente para delimitar las áreas de distribución de las especies a esta escala local, los análisis de selección de reservas pueden basarse en las probabilidades de distribución obtenidas tras la realización de modelos predictivos de distribución individuales (Austin, 2002).

Los resultados de un proceso de selección como este deben mejorarse también incorporando la probabilidad de persistencia de las distintas poblaciones (Araújo \& Williams, 2000) y, cuando exista información suficiente, considerando la variabilidad intraespecífica de los taxones, de modo que sean seleccionadas aquellas áreas con poblaciones genéticamente diferenciadas. La información biológica de partida es, como ocurre casi siempre, el punto clave para el éxito de estos procesos de selección (Freitag et al., 1996). Por ejemplo, en el presente ejemplo los valores de rareza de las especies han sido calculados teniendo en cuenta el territorio ocupado sobre el total Ibérico y, es evidente, que una especie puede ser rara en nuestra península pero común en otras latitudes próximas. Este inconveniente puede solucionarse en gran medida, asignando a cada especie un valor de conservación específico previamente convenido, o determinado estos valores de conservación según la tolerancia y/o la especialización ambiental de cada una de las especies para el territorio deseado. Estos requisitos de conservación específicos pueden ser considerados mediante la realización de procesos de selección de reservas con programación linear (Rodrigues \& Gaston, 2002; ver Carrascal \& Lobo, 2003 para un ejemplo reciente de esta técnica en nuestra península).

En conclusión, las aproximaciones científicas dirigidas a la selección de áreas de conservación han adquirido un notable desarrollo y tenemos a nuestra disposición herramientas fiables y eficaces con capacidad para delimitar una red de reservas que garantice la representación de un conjunto de organismos. Sin embargo, esas aproximaciones apenas son utilizadas y conocidas (Prendergast, 1999). Tal vez, el encuentro entre los taxónomos especialistas de cada grupo y los biólogos de la conservación conocedores de estas técnicas, permita superar el alejamiento existente entre los encargados de elaborar las propuestas de conservación y los investigadores.

\section{AGRADECIMIENTOS}

Este trabajo ha sido posible gracias a los proyectos REN2001-1136/GLO del MCyT y 07M/0080/2002 de la Comunidad de Madrid, así como al "Improving Human Potential Programme” BIOD-IBERIA.

\section{Referencias}

Andriamampianina, L., Kremen, C., Vane-Wright, D., LEes, D. \& RAZAFimahatratra, V., 2000. Taxic richness patterns and conservation evaluation of Madagascan tiger beetles (Coleoptera: Cicindelidae). Journal of Insect Conservation, 4: 109-128.

AraúJo, M. B., 1999. Distribution patterns of biodiversity and the design of a representative reserve network in Portugal. Diversity and Distributions, 5: 151-163.

Araújo, M. B., Humphries, C. J., Densham, P. J., Lampinen, R., HagemeiJer, W. J. M., MitchellJONES, A. J. \& GASC, J. P,. 2001. Would environmental diversity be a good surrogate for species diversity? Ecography, 24: 103-110.

AraúJo, M. B. \& Williams, P. H., 2000. Selecting areas for species persistence using occurrence data. Biological Conservation, 96: 331-345.

Austin, M. P., 2002. Spatial prediction of species distribution: an interface between ecological theory and statistical modelling. Ecological Modelling, 157: 101-118.

Buckland, S. T. \& Elton, D. A., 1993. Empirical models for the spatial distribution wildlife. Journal of Applied Ecology, 30: 478-495.

Carrascal, L. M. \& Lobo, J. M., 2003. Respondiendo viejas preguntas con nuevos datos: Una aproximación al estudio de los grandes patrones de la avifauna española y sus consecuencias para la conservación. En: Atlas y Libro Rojo de la Aves de España. Ministerio de Medio Ambiente. Madrid: 651-668.

Csuti, B., Polasky, S., Williams, P. H., Pressey, R. L., Camm, J. D., Kershaw, M., Kiester, A. R., Downs, B., Hamilton, R., Huso, M. \& SAhr, K., 1997. A comparison of reserve selection algorithms using data on terrestrial vertebrates in Oregon. Biological Conservation, 80: 83-97.

Faith, D. P. \& W alker, P. A., 1996. Environmental diversity: on the best-possible use of surrogate data for assessing the relative biodiversity of sets of areas. Biodiversity and Conservation, 5: 399-415.

Freitag, S., Nicholls, A. O. \& van JaARsveld, A. S., 1996. Nature reserve selection in the Transvaal, South Africa: what data should we be using? Biodiversity and Conservation, 5: 685-698.

Gasc, J. P., Cabela, A, Crnobrnja-Isailovic, J., Dolmen, D., Grossenbacher, K., Haffner, P., 
Lescure, J., Martens, H., Martinez Rica, J. P., Maurin, H., Oliveira, M. E., Sofianidou, T. S., Veith, M \& ZuiderwiJk, A. (eds.), 1997. Atlas of Amphibians and Reptiles in Europe. Societas Europaea Herpetologica \& Muséum National d'Histoire Naturelle (IEGB/SPN). Paris. 496 pp.

Howard, P. C., Viskanic, P., DAVEnPort, T. R. B., Kiegnyi, F. W., Baltzer, M., Dickinson, C. J., Lwanga, J. S., Matthews, R. A. \& Balmford, A., 1998. Complementarity and the use of indicator groups for reserve selection in Uganda. Nature, 394: 472-475.

Kershaw, M, Williams, P. H. \& Mace, G. C., 1994. Conservation of Afrotropical antelopes: consequences and efficiency of using different site selection methods and diversity criteria. Biodiversity and Conservation, 3: 354-372.

Kier, G. \& BARTHLOTT, W., 2001 Measuring and mapping endemism and species richness: a new methodological approach and its application on the flora of Africa. Biodiversity and Conservation, 10: 1513-1529.

Margules, C. R., Nicholls, A. O. \& Pressey, R. L. 1988. Selecting networks of reserves to maximize biological diversity. Biological Conservation, 43: 63-76.

Margules, C. R. \& Pressey, R. L., 2000. Systematic conservation planning. Nature, 405: 243-253.

Mateo, J. A., 2002. Áreas importantes para la herpetofauna española. En: Atlas y libro rojo de los anfibios y reptiles de España. J. M. Pleguezuelos, R. Marquez \& M. Lizana (eds.). Dirección General de Conservación de la Naturaleza. Madrid: 483-500.

Montaña, E. \& Rey Benayas, J. M., 2002. ¿Coinciden los espacios naturales protegidos con las áreas relevantes de diversidad de herpetofauna en España peninsular y Baleares? Ecosistemas 2002/2 (URL: http//www.aeet.org/ecosistemas/022/investigacion2.htm).

Pleguezuelos, J. M. (ed.), 1997. Distribución y biogeo grafia de los anfibios y reptiles de España y Portugal. Monografías de Herpetología, vol. 3, Asociación Herpetológica Española. Granada. 542 pp.

Pleguezuelos, J. M., 2002. Las especies introducidas de anfibios y reptiles. En: Atlas y libro rojo de los anfi bios y reptiles de España. J. M. Pleguezuelos, R. Marquez \& M. Lizana (eds.). Dirección General de Conservación de la Naturaleza. Madrid: 501-529.

Pleguezuelos, J. M., Marquez, R. \& Lizana, M. (eds.), 2002. Atlas y libro rojo de los anfibios y reptiles de España. Dirección General de Conservación de la Naturaleza-Asociación Herpetológica Española. Madrid. 584 pp.
Prendergast, J. R., Quinn, R. M. \& Lawton, J. H., 1999. The gaps between theory and practice in selecting nature reserves. Consenation Biology, 13: 484-492.

Pressey, R. L., Humphries, C. J., Margules, C. R., VAne-Wright, R. I. \& Williams, P. H., 1993. Beyond opportunism: key principles for systematic reserve selection. Trends in Ecology and Evolution, 8: $124-128$.

Reyers, B., van JaARsveld, A. S. \& KrÜGer, M., 2000. Complementarity as a bioindicator strategy. Proceedings of the Royal Society of Londond, Series $B, 267: 505-513$.

Ricklefs, R. E. \& Schluter, D. (eds.), 1993. Species diversity in ecological communities. The university of Chicago Press. Chicago. 416 pp.

Rodrigues, A. S. L. \& GAston, K. J., 2002. Optimisation in reserve selection procedures-why not? Biological Conservation, 107: 123-129.

SAlvador, A., 1998. Reptiles. Fauna Ibérica vol. 10, Consejo Superior de Investigaciones Científicas. Madrid. 705 pp.

SAlVAdor, A. \& GARcía PARís, M., 2001. Anfibios espa ñoles. Identificación, historia natural y distribución. Canseco. Talavera de la Reina. 269 pp.

SimBerlofF, D., 1998. Flagships, umbrellas, and keystones: is single-species management passe in the landscape era? Biological Conservation, 83:247-257.

Turpie, J. K., Beckley, L. E. \& KatuA, S. M., 2000. Biogeography and the selection of priority areas for conservation of South African coastal fishes. Biological Conservation, 92: 59-72.

Williams, P., Gibbons, D., Margules, C., Rebelo, A., Humphries, C. \& Pressey, A. R., 1996. Comparison of richness hotspots, rarity hotspots and complementary areas for conserving diversity using British birds. Conservation Biology, 10: 155-174.

Williams, P. H., 1999. Worldmap 4.1 Windows: softwa re and user document 4. Privately distributed. London. http://www.nhm.ac.uk/science/projects/worldmap (no publicado).

Williams, P. H., Burgess, N. D. \& RahbeK, C., 2000. Flagship species, ecological comnplementarity and conserving the diversity of mammals and birds in subSaharan Africa. Animal Conservation, 3: 249-260. 